\title{
Diferencias demográficas de climas motivacionales, necesidades psicológicas básicas y cohesión en jóvenes \\ Demographic differences in motivational climates, basic psychological needs and cohesion in young people
}

*Sofia Mosqueda, *Luis T. Ródenas-Cuenca, **Isabel Balaguer, *Yadira Elizabeth Salcido Otañez, *Jeanette M. López-Walle

*Universidad Autónoma de Nuevo León (México), **Universidad de Valencia (España)

Resumen: El objetivo de este trabajo es analizar el comportamiento de las variables de clima motivacional (empowering y disempowering), necesidades psicológicas (satisfacción y frustración) y cohesión (tarea y social) en función del sexo (hombres y mujeres), naturaleza de la actividad física (deporte conjunto-individual y educación física) y años de experiencia deportiva (4 años o menos, 5 años o más); además, analizar la interrelación entre las variables psicológicas. Participaron 237 jóvenes deportistas mexicanos $($ Medad $=12.22, \mathrm{DT}=1.27)$ de nivel básico. Los resultados muestran que: 1$)$ respecto al género no hay diferencias significativas; 2) considerando la naturaleza de la actividad, hay diferencia en todas las variables psicológicas; y 3) por años de experiencia, únicamente el clima empowering mostró diferencias significativas, siendo mayor en los deportistas con menos experiencia. Respecto a la interacción entre variables, se evidencia la relación positiva entre el clima empowering, la satisfacción de las NPB y ambas cohesiones; así como, el clima disempowering y la frustración de las NPB. Para poder ser concluyentes en estos resultados es necesario realizar una mayor investigacion.

Palabras claves: climas motivacionales, necesidades psicológicas básicas, cohesión, variables demográficas.

\begin{abstract}
Abtract: The objective of this work is to analyze the behavior of the variables of motivational climate (empowering and disempowering), psychological needs (satisfaction and frustration) and cohesion (task and social) as a function of sex (men and women), nature of physical activity (group-individual sport and physical education) and years of sport experience (4 years or less, 5 years or more); in addition, to analyze the interrelation between the psychological variables. A total of 237 young Mexican athletes (Mage $=12.22, \mathrm{SD}=1.27$ ) of elementary level participated. The result show that: 1 ) respect to the differences according to gender there were no significant differences; 2) with respect to the nature of the activity, there were differences between all the psychological variables; 3) according to the years of experience, only the empowering climate showed a significant difference, being greater in the athletes with fewer years of experience. Regarding the interaction between variables, there was evidence of a positive relationship between the empowering climate, NPB satisfaction and both cohesions, as well as the disempowering climate and NPB frustration. In order to be conclusive about these results, further research is needed.
\end{abstract}

Keyword: motivational climates, basic psychological needs, cohesion, demographic variables.

\section{Introducción}

Los climas motivacionales bajo el modelo de Duda, Appleton, Stebbings y Balaguer (2018) fusionan la teoría de las metas de logro (Achievement Goal Theory, AGT, Ames, 1992; Nicholls, 1984) y la teoría de la autodeterminación (Self-Determination Theory, SDT, Ryan \& Deci, 2017) en un modelo jerárquico y multifactorial, destacando cómo las acciones del entrenador afectan directamente la calidad de la motivación y los indicadores de bienestar psicológico de los deportistas.

Dentro de este modelo se conceptualizan los climas

Fecha recepción: 09-03-20. Fecha de aceptación: 05-08-21

Sofia Mosqueda

smo94g@hotmail.com motivacionales en dos vertientes; clima empowering y clima disempowering. El clima empowering es un clima motivacional que se caracteriza por una alta implicación en la tarea, alto apoyo a la autonomía y alto apoyo social, lo que le ayudará al deportista, entre otras cosas, a manifestar indicadores de funcionamiento óptimo. Por otro lado, en el clima disempowering el entrenador utiliza un estilo controlador y crea climas de alta implicación en el ego.

Los climas empowering y disempowering se han relacionado con las Necesidades Psicológicas Básicas (NPB), siendo así que, el clima empowering facilita la satisfacción de las NPB (Castillo, López-Walle, Tomás, \& Balaguer, 2017; Hancox, Quested, Ntoumanis, \& Duda, 2017) y esto conlleva a un funcionamiento óptimo y bienestar psicológico (Duda \& Appleton, 2016); 
mientras que, el clima disempowering favorece la frustración de las NPB (Chu, 2018; Ramírez, 2020), lo cual conlleva un funcionamiento comprometido y malestar (Erlend, Ivarsson, Haug, \& Ommundsen, 2018). Una revisión sistemática realizada por Salazar-Ayala y Gastélum-Cuadras (2020) evidenció la influencia del entrenador sobre las NPB, además muestra la falta de información con respecto a la frustración de las NPB en poblaciones de educación física de Latinoamérica.

La NPB son extraídas de la teoría de la autodeterminación (Ryan \& Deci, 2000, 2017) y son definidas como un nutriente psicológico esencial para la adaptación, la integridad y el crecimiento de los individuos, se consideran tres necesidades: autonomía, que es el sentimiento de dar dirección a las propias acciones y conductas; competencia, es el sentido de efectividad, eficacia y dominio; y la relación, que es la necesidad de sentirse interrelacionados con otros de forma significativa, creando vínculos de cuidado, cariño, atención y reciprocidad.

Así mismo, las NPB se han relacionado con la cohesión, siendo así que la cohesión social se relaciona positivamente con la satisfacción general de las NPB (Erikstad, Martin, Haugen, \& Høigaard, 2018; Heuzé, Eys, Dubuc, Bosselut, \& Couture, 2018).

La cohesión se define como «un proceso dinámico que refleja la tendencia del grupo a mantenerse y permanecer unido en la búsqueda de sus objetivos instrumentales y/o para la satisfacción de las necesidades afectivas de sus miembros» (Carron, Widmeyer, \& Brawley, 1985, p. 245). Se plantea que esta puede estar orientada hacia dos factores: orientación tarea, que es el grado en el que un grupo permanece unido debido a la búsqueda de cumplir objetivos instrumentales; y orientación social, grado en el que los miembros del grupo permanecen unidos debido al disfrute del compañerismo.

Las variables anteriormente mencionadas (clima motivacional empowering y disempowering, NPB y cohesión) han demostrado diferencias entre características descriptivas tales como el sexo, la naturaleza de la actividad física (AF) y la experiencia, las cuales se mencionarán a continuación.

Respecto al sexo, los climas empowering y disempowering no se ha encontrado artículos cuyo objetivo comparen directamente las variables entre hombres y mujeres, sin embargo, en los pocos artículos que se reportan las medias diferenciando hombres y mujeres se observa que son mayores los puntajes obtenidos en los hombres en ambos tipos de climas (Chu, 2018). Con relación a las NPB, se ha observado que los hom- bres tienen niveles más altos de satisfacción en comparación con las mujeres, además, estas presentan niveles inferiores de frustración de las NPB (Chu, 2018); en esta misma línea, un estudio por Parra-Plaza, VílchezConesa y De Francisco-Palacios (2018) muestra que los hombres tienen mayores niveles de satisfacción de la autonomía y mayores niveles de la satisfacción de relación, mismos resultados que son apoyados por Leytón, García, Fuentes y Jiménez (2018); pero diferente a lo mostrado por Amado, Sánchez-Miguel, Leo, SánchezOliva y García-Calvo (2014) en donde no hay diferencias significativas. En cuanto a la cohesión, las mujeres suelen tener mayores niveles de cohesión orientada la tarea en comparación con los hombres, lo que implica que las mujeres muestran mayor implicación en la búsqueda de los objetivos de equipo en comparación con los varones (Nascimento Jr, Silva, Granja, Oliveira, \& Fortes, 2019)

Respecto a la naturaleza de la $A F$, en los climas empowering y disempowering no se encontró literatura; sin embargo, se ha observado que los climas motivacionales varían con respecto a la naturaleza de la AF (Castro-Sánchez, Zurita-Ortega, \& Chacón-Cuberos, 2018; Cordo-Cabal, Gómez-López, Granero-Gallegos, \& Sánchez-Alcaraz, 2019) y al comparar los datos de diferentes estudios encontramos una variación descriptiva, por ejemplo, los deportes de conjunto obtienen puntuaciones más altas del clima empowering (Gutiérrez-García, López-Walle, Tomás, Tristán, \& Balaguer, 2019) comparado con otro estudio en deportes individuales (Romina, 2019). Considerando a las NPB, en una revisión sistemática realizada por García (2016) se observó que los deportistas colectivos presentan mayores niveles en la satisfacción de la necesidad de relación, mientras que, los deportistas individuales presentan niveles superiores en la necesidad de autonomía; en el mismo estudio, se evidencia la falta de literatura con respecto al comportamiento de la cohesión en deportes individuales y muestra como la cohesión tarea es una variable fundamental para el desarrollo del rendimiento en deportes de conjunto, mientras que, la variable de cohesión social debe de ser tratada con reserva, ya que niveles muy bajos o muy altos se relacionan con bajos niveles de rendimiento, esto igualmente en deportes de conjunto. Una revisión sistemática realizada por Salazar-Ayala \& Gastélum-Cuadras (2020) evidencia la falta de producción científica con respecto a la frustración de las necesidades psicológicas básicas y la educación física en poblaciones latinoamericanas.

Respecto a la experiencia deportiva, se presentan pun- 
tuaciones más altas de clima empowering en los deportistas con mayor experiencia, los cuales participan en competencias nacionales e internacionales y/o cuentan con más de 4 años en el deporte (Mosqueda, LópezWalle, Gutiérrez-García, García-Verazaluce, \& Tristán, 2019); comparado con los valores de otro estudio que incluyó deportistas con menos de 4 años antigüedad (Ramírez, 2020). Considerando las NPB, en un estudio realizado por Parra-Plaza et al. (2018) mencionan que la satisfacción de la competencia y autonomía aumentan conforme los años de experiencia. En cuanto a la cohesión, no se encontró literatura, pero al comparar diferentes artículos encontramos que la cohesión tanto tarea como social presenta descriptivamente niveles superiores en deportistas novatos, menos de 5 años de experiencia (Nascimiento Jr. et al., 2017) en comparación con los deportistas experimentados, 5 años o más (Dobrijeviæ, Đorðeviæ Boljanoviæ, Alèakoviæ, \& Lazareviæ, 2020).

Con base a la evidencia empírica mencionada anteriormente, se observa escasa literatura en psicología diferencial, la mayoría de los estudios se enfocan principalmente a las relaciones entre variables en poblaciones homólogas o únicas. Por lo que, resulta importante indagar las posibles diferencias en función de las características de las poblaciones que realizan AF con respecto a las variables psicológicas que suelen interaccionar. Por lo tanto, se plantearon dos objetivos en este trabajo; primero, analizar el comportamiento de las variables de clima motivacional (empowering y disempowering), necesidades psicológicas (satisfechas y frustradas) y cohesión (tarea y social) en función del sexo, naturaleza de la AF y experiencia deportiva; y segundo, analizar la interrelación entre las variables psicológicas, teniendo como hipotesis que; 1) el clima empowering relacionará de forma positiva y significativa con la satisfacción de las necesidades psicológicas básicas y la cohesión tanto tarea como social y negativamente con la frustración de las necesidades psicológicas básicas; 2) el clima disempowering relacionará positivamente con la frustración de las necesidades psicológicas básicas y negativamente con la cohesión tarea y social.

\section{Materiales y Métodos}

\section{Participantes}

Participaron 237 deportistas pertenecientes a equipos deportivos de un colegio privado de nivel básico $\left(6^{\circ}\right.$ de primaria y de $1^{\circ}$ a $3^{\circ}$ de secundaria). El rango de edad oscila desde 12 a 17 años $(M=12.22, D T=1.27)$, de los cuales, 118 eran hombres y 119 mujeres; con base a la naturaleza de la AF, 156 deportistas pertenecen a deportes de conjunto (futbol y baloncesto), 35 deportistas a deportes individuales (atletismo y natación), y 46 a la clase de educación física; con respecto a la experiencia deportiva; 108 deportistas contaban con cuatro años o menos de antigüedad en su deporte y 129 deportista con más de cinco años.

\section{Instrumentos}

Para medir la percepción de los climas motivacionales empowering y disempowering se utilizó la versión mexicana del cuestionario Clima Motivacional Empowering y Disempowering (Empowering and Disempowering Motivational Climate Questionnaire-Coach, EDMCQ-C; Appleton, Ntoumanis, Quested, Viladrich, \& Duda, 2016) utilizado en el contexto mexicano (Castillo et al., 2017; Mosqueda et al., 2019), el cual cuenta con 32 ítems divididos en cinco subescalas de primer orden, referidas a conductas de: implicación en la tarea, formado por 9 ítems (e.g. «Mi entrenador anima a los jugadores a intentar/probar nuevas habilidades»), apoyo a la autonomía, con 5 ítems (e.g. «Mi entrenador ofrece/otorga a los jugadores alternativas y opciones»), apoyo social, formado por 3 ítems (e.g. «Mi entrenador realmente aprecia a los jugadores como personas no sólo como atletas»), implicación en el ego, con 7 ítems (e.g. «Mi entrenador cambia a los jugadores cuando ellos han cometido un error»), y estilo controlador, con 8 ítems (e.g. «Mi entrenador presta menos atención a los jugadores si ellos le disgustan/desagradan»). Las tres primeras subescalas de primer orden se promedian para generar una dimensión de segundo orden denominada clima empowering; mientras que las dos últimas subescalas se promedian para generar otra dimensión de segundo orden denominada clima disempowering. Las respuestas se recogen en una escala tipo Likert de cinco puntos que oscila desde Totalmente en desacuerdo (1) hasta Totalmente de acuerdo (5). Esta escala ha presentado adecuadas propiedades psicométricas en sus dimensiones de empowering y disempowering (Appleton et al., 2016; Chu, 2018).

Para evaluar la satisfacción de las NPB se utilizó el promedio de los valores obtenidos en tres instrumentos:

- La satisfacción de la autonomía se evaluó mediante la Escala de Autonomía Percibida en el Deporte (Perceived Autonomy in Sport Scale, PASS; Reinboth y Duda, 2006), utilizando la versión mexicana (López-Walle, 
Balaguer, Castillo, \& Tristán, 2012). La escala consta de 10 ítems (e.g. «Yo puedo dar mi opinión»), con un tipo de respuesta Likert de siete puntos que oscila entre (1) totalmente en desacuerdo a (7) totalmente de acuerdo. Esta escala ha presentado adecuadas propiedades psicométricas (González, Castillo, García-Merita, \& Balaguer, 2015; López-Walle et al., 2012).

- La satisfacción de la competencia se midió mediante la Escala de Competencia Percibida del Inventario de Motivación Intrínseca (Intrinsic Motivation Inventory, IMI; McAuley, Duncan, \& Tammen, 1989) en su versión adaptada al contexto mexicano de López-Walle et al. (2012). Esta escala está compuesta por cinco ítems (e,g «Soy bastante hábil en mi deporte»), el tipo de respuesta es Likert de siete puntos que oscila entre (1) totalmente en desacuerdo a (7) totalmente de acuerdo. Esta escala ha presentado adecuadas propiedades psicométricas (González et al., 2015; López-Walle et al., 2012).

- La satisfacción de la relación se evaluó mediante la subescala de Aceptación de la Escala de Necesidades de Relación (Perceived Relatedness Scale, PRS; Richer y Vallerand, 1998) mediante la versión mexicana (LópezWalle et al., 2012), conformada por cinco ítems (e.g «me siento apoyado(a)»), con escala de respuesta tipo Likert de cinco puntos que oscilan entre (1) totalmente en desacuerdo a (5) totalmente de acuerdo. Esta escala ha presentado adecuadas propiedades psicométricas (González et al., 2015; López-Walle et al., 2012).

Para evaluar la frustración de las NPB, se empleó la Escala de la Frustración de las NPB (Psychological Need Thwarting Scale, PNTS; Bartholomew, Ntoumanis, Ryan, Bosch, \& Thøgersen-Ntoumani, 2011) versión adaptada al contexto mexicano (Cantú-Berrueto, Castillo, LópezWalle, Tristán, \& Balaguer, 2016). La escala está conformada por 12 ítems divididos en 3 subescalas, las cuales son: Frustración de la necesidad de autonomía con 4 ítems (e.g. «Siento que se me impide tomar decisiones respecto a mi forma de entrenar en mi deporte»), frustración de la necesidad de competencia con 4 ítems (e.g. «En mi deporte hay situaciones que me hacen sentir incapaz), y frustración de la necesidad de relación con 4 ítems (e.g. «En mi deporte siento que los demás no me tienen en cuenta). Las respuestas se recogen en una escala tipo Likert de cinco puntos que oscila desde Totalmente en desacuerdo (1) hasta Totalmente de acuerdo (5). Esta escala ha presentado adecuadas propiedades psicométricas en sus dimensiones (Cantú-Berrueto et al., 2016; Mars, Castillo, López-Walle, \& Balaguer, 2017).
Para evaluar la cohesión de equipo, se utilizó el Cuestionario de Entorno Deportivo para Jóvenes (The Youth Sport Environment Questionnaire, YSEQ; Eys, Loughead, Bray, \& Carron, 2009) en su versión al castellano de Castillo, Rodenas, López-Walle, \& Balaguer (2017). El cuestionario está compuesto por 18 ítems divididos en dos subescalas: cohesión de tarea, con 8 ítems (e.g «Todos hemos estado igual de comprometidos con los objetivos del equipo») y la cohesión social, con 8 ítems (e.g «Algunos de mis mejores amigos están en este equipo»). El tipo de respuesta es Likert de cinco puntos que oscila desde Muy en desacuerdo (1) hasta Muy de acuerdo (5). Esta escala ha presentado adecuadas propiedades psicométricas en sus dos dimensiones (Castillo et al., 2017; Moehnke, 2019).

\section{Procedimiento}

Se obtuvo el consentimiento informado de participación de los entrenadores del colegio privado y de sus respectivos atletas. Las aplicaciones se realizaron de forma grupal antes de iniciar el entrenamiento de cada uno de los equipos participantes en aulas de clases previamente asignadas, al comenzar las sesiones se aplicaron los cuestionarios vía electrónica (SurveyMonkey) a través de tabletas. Durante la aplicación de los cuestionarios estaban presentes al menos dos investigadores capacitados para resolver dudas y verificar su correcta aplicación, además a los deportistas se les informó el propósito de la investigacion, así como de la confidencialidad de su información, remarcando que la participación era voluntaria, por lo que podrían retirarse en cualquier momento sin ninguna consecuencia negativa hacia ellos. La aplicación de los cuestionarios duraba aproximadamente $15 \mathrm{~min}$ y se realizaron fuera de la etapa competitiva en un periodo aproximado de 2 semanas.

\section{Análisis estadístico}

Para dar respuesta a los dos objetivos del estudio; en primer lugar, se realizó el análisis descriptivo (media, mínimo, máximo y desviación típica) de las variables psicológicas y la fiabilidad de los instrumentos utilizados para medirlas, este análisis a través del alfa de Cronbach; en segundo lugar, se realizaron correlaciones entre las diferentes variables psicológicas de estudio para analizar su interacción; en tercer lugar, se utilizó el análisis MANOVA para encontrar diferencias del comportamiento de las variables psicológicas en función del sexo, naturaleza de la AF y experiencia deportiva; en cuarto lugar, se realizaron ANOVAs y pruebas $t$ para discrimi- 
nar las variaciones especificas con respecto a las variables psicológicas. Por último, para conocer el tamaño del efecto entre las diferencias significativas se utilizó la d de Cohen.

Todos los análisis se realizaron mediante el software IBM SPSS STATISTICS 21, y específicamente los tamaños del efecto mediante el siguiente enlace https: / / www. campbellcollaboration.org/ escalc/html/ EffectSizeCalculator-SMD5.php.

\section{Resultados}

En la Tabla 1 se muestran los análisis descriptivos de las variables psicológicas considerando toda la muestra, así como la fiabilidad de los instrumentos que las miden y las correlaciones entre las variables; en la Tabla 2 se muestra el resultado del análisis MANOVA y en las Tablas 3, 4 y 5 se observan los análisis de las pruebas $t$ y ANOVA en función de las características a comparar, como el sexo, naturaleza de la actividad y experiencia en el deporte, además de los respectivos estadísticos de contraste.

$\mathrm{Al}$ analizar las correlaciones entre las variables utilizadas (Tabla1), los resultados muestran correlaciones significativas y positivas del clima empowering con la satisfacción de las NPB, la cohesión tarea y social y ne-

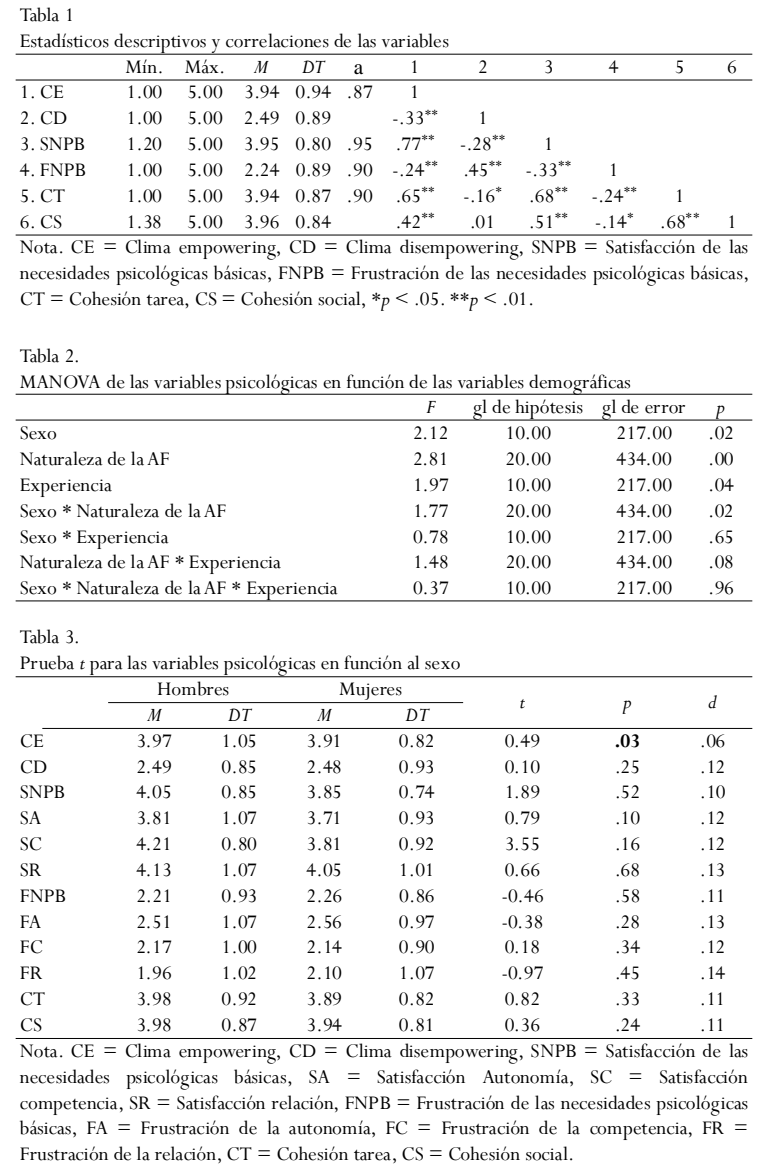

gativamente con la frustración de las NPB; mientras que, el clima disempowering se relacionó significativa y negativamente con la satisfacción de las NPB y ambas cohesiones, y de forma positiva con la frustración de las NPB. Además, la satisfacción de las NPB relaciona de forma positiva y significativa con ambas cohesiones; mientras que, la frustración de las NPB lo hace de forma negativa.

Al analizar las diferencias en función de las características de la población se presentan en la Tabla 2 los resultados del MANOVA en donde se tomaron como variables dependientes el clima empowering, clima disempowering, satisfacción de las NPB, frustración de las NPB, cohesión tarea y cohesión social, y como variables independientes el sexo, naturaleza de la AF y experiencia deportiva. Dentro de los resultados observamos que el sexo, naturaleza de la actividad física y experiencia deportiva por sí mismas afectan el comportamiento de las variables psicológicas al mostrar significancia, sin embargo, al tomarlas en cuenta en su conjunto no se obtienen diferencias significativas con excepción del sexo en conjunto con la naturaleza de la $\mathrm{AF}$.

$\mathrm{Al}$ analizar las variables de forma individual se encontró lo siguiente: 1) en función al sexo (Tabla 3), no se encontraron diferencias significativas entre las variables

Tabla 4 .

ANOVA y post hoc para las variables psicológicas en función con la naturaleza de la AF

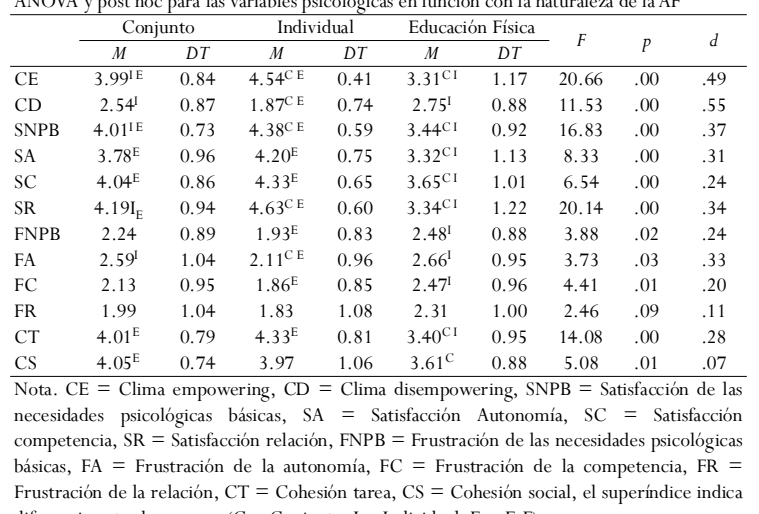

diferencia entre los grupos $(\mathrm{C}=$ Conjunto, $\mathrm{I}=$ Individual, $\mathrm{E}=\mathrm{E} . \mathrm{F})$.

Tabla 5.

Prueba $t$ para las variables psicológicas en función a la experiencia deportiva

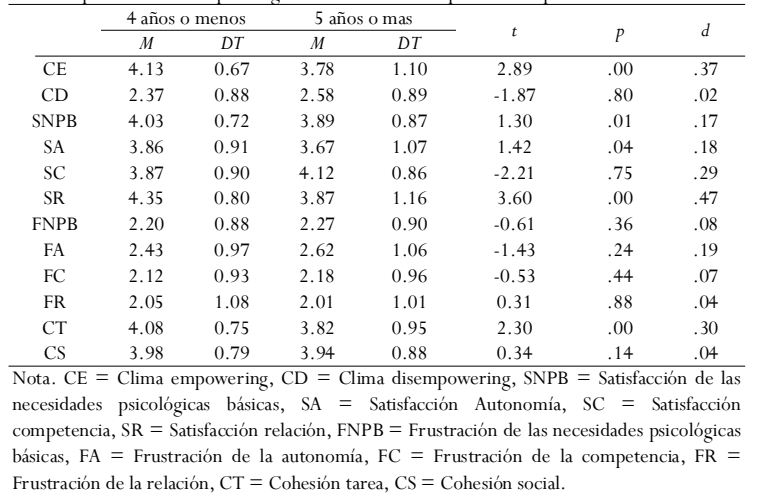


psicológicas excepto en el clima empowering, donde los hombres presentan niveles superiores aunque el tamaño de efecto es pequeño: 2) en función a la naturaleza de la AF (Tabla 4), se presenta una diferencia significativa entre los tres grupos (deporte de conjunto, individual y EF) en todas las variables psicológicas con excepción de la frustración de la relación, dentro de las variables donde hubo diferencias el deporte individual obtiene las mayores puntuaciones con excepción de la cohesión social donde el deporte de conjunto puntúa mayor, observamos un tamaño de efecto medio bajo en todas las variables significativas; y, 3) para analizar las diferencias significativas en función de la experiencia (Tabla 5) se comprobó que existe diferencia significativas en clima empowering, satisfacción de las NPB y satisfacción de la necesidad de autonomía, satisfacción de la relación y cohesión tarea, en estas los sujetos que tienen menos de 4 años de experiencia puntúan más alto y observamos un tamaño de efecto medio bajo.

\section{Discusión}

Uno de los objetivos de este trabajo fue analizar el comportamiento de las variables de clima motivacional empowering y disempowering, necesidades psicológicas (satisfechas y frustradas) y cohesión (tarea y social) en función del sexo, naturaleza de la AF y experiencia deportiva; asimismo, analizar la interrelación entre las variables psicológicas.

De acuerdo con los resultados obtenidos por el MANOVA al analizar las tres variables demográficas (sexo, naturaleza de la actividad física y experiencia deportiva) no se observan diferencias significativas, esto pudiéndose deber al contexto en el cual se esta evaluando, ya que se efectuó en un contexto de AF por la salud, donde sin importar los años de experiencia o el tipo de actividad física a practicar el principal enfoque es la recreación y la salud por lo que esto pudiese estar influenciando el carácter de los objetivos, así como la presión hacia los deportistas y su forma de interacción con los entrenadores y compañeros, sin embargo a raíz de la falta de literatura dentro de este tema sería necesario realizar mayor investigacion para obtener una conclusión.

Por otra parte al contrastar directamente las variables demográficas se observa que únicamente hay una diferencia significativa en el comportamiento de las variables psicológicas al tomar en cuenta tanto el sexo como la naturaleza de la AF, si bien no hemos encontrado estudios con los cuales poder comparar estos resulta- dos en concreto, si se ha observado que la selección del tipo de AF a practicar puede depender del sexo y de los estereotipos y la conotación social que acompaña a este, se ha observado que los hombres demandan más deportes colectivos y competitivos mientras que las mujeres prefieren los deportes individuales orientados hacia la salud (Pavón \& Moreno, 2008), pudiendo ser que estas diferencias en cuanto a preferencias sea lo que marca el cambio de comportamiento en las variables psicológicas estudiadas en este trabajo, sin embargo, esto debe de tomarse con cautela debido a la falta de evidencias empíricas.

Siguendo con los resultados específicos en función de las variables demográficas encontramos que con respecto al sexo, al comparar el comportamiento de las variables psicológicas, los resultados no mostraron diferencias significativas, excepto en el clima empowering, favoreciendo a los hombres, este último coincide con la literatura (Chu, 2018; Cordo-Cabal et al., 2019; Torregrosa, Viladrich, Ramis, Azócar, Latinjak, \& Cruz, 2011); sin embargo, estos mismos estudios si encontraron diferencias significativas en función del sexo en el clima disempowering, donde los hombres tenían mayores puntuaciones, en este estudio la diferencia no fueron significativas estadísticamente pero los hombres tiene una puntuación levemente mayor que las mujeres.

Con respecto a la satisfacción de las NPB, nuestros resultados difieren parcialmente de lo encontrado en un estudio realizado por Leytón et al. (2018) donde se observa que los hombres tiene una mayor satisfacción de la autonomía mientras que las mujeres presentan una mayor satisfacción de la relación, en la satisfacción de la competencia no se encontraron diferencias significativas, cabe señalar que este estudio fue realizado en una población de 18 a 64 años; por otro lado, en un estudio realizado por Amado et al. (2014) en una población con edades comprendidas entre 11 y 16 años coinciden con nuestros resultados, confirmando que no hay diferencias significativas en la satisfacción de la necesidad de autonomía ni en la de relación. En cuanto a la frustración de la NPB no se encontraron estudios que las analicen desde esta perspectiva, por lo que nuestros resultados resultan innovadores, resaltando que no existe diferencia significativa entre hombres y mujeres dentro de la frustración de autonomía, competencia y relación.

Con respecto la cohesión grupal se observa una discrepancia dentro de la literatura, un estudio realizado con deportes de equipo indica que la cohesión tarea difiere entre el sexo, siendo mayor en las mujeres 
(Nascimento Jr et al., 2019); sin embargo, en otro estudio realizado igualmente con deportistas de conjunto no se encontraron diferencias significativas en la cohesión tarea (Ferrnández, 2016). Los resultados de este estudio coinciden con el segundo estudio de Ferrnández (2016), en donde no se reportan diferencias significativas con respecto a el sexo.

Las diferencias en los resultados pueden deberse a dos factores, en primer lugar, la literatura se enfoca principalmente en el ámbito deportivo, y dentro de este, la mayoría en deportes de conjunto; por lo que este estudio aporta información para otro ámbito, como la educación física, así como dentro del ámbito deportivo, se considera tanto el deporte en conjunto como el individual. En segundo lugar, y la cual podría tener mayor relevancia, los sujetos evaluados en la literatura tienen medias de edad superior a la de este estudio (12 años), lo que implica que aún están en proceso de maduración biopsicosocial al encontrarse en el inicio de la adolescencia, ya que en esta etapa es donde se desarrollan las principales diferencias físicas, cognitivas y emocionales entre hombres y mujeres (Papalia, Olds, \& Feldman, 2009); por lo tanto, la edad podría ser un factor que esté modificando el comportamiento y apreciación de estas variables psicológicas, sin embargo, para poder ser concluyentes se requieren mayores estudios.

En función de la naturaleza de la $A F$ encontramos resultados interesantes, ya que se observaron diferencias significativas dentro de todas las variables psicológicas y es poca la literatura encontrada, sobre todo conforme a la diferencia entre el ámbito deportivo y la educación física.

Con respecto a el clima empowering se observó diferencia en el comportamiento entre los tres grupos (deporte de conjunto, individual y educación física), esta diferencia es apoyada por otros artículos (Castro-Sánchez et al., 2018; Cordo-Cabal et al., 2019), sin embargo, en el clima disempowering no se observa una diferencia directa entre el deporte de conjunto y la educación física. Esto mismo se observa en la frustración general de las NPB donde no se muestra una diferencia entre el deporte de conjunto y la educación física, esto puede deberse al componente de equipo y socialización existente en ambos tipos de actividad; sin embargo, poder explicar este fenómeno sería necesario investigar más a fondo las diferencias y/o similitudes a nivel psicológico entre el deporte de conjunto y la educación física, ya que en la literatura revisada no se encontró evidencia que lo aborde en las variables utilizadas en este estudio.

En cuanto a las satisfacciones de la NPB de forma individual, observamos que la autonomía, competencia y relación, muestran diferencias significativas entre el deporte, tanto conjunto como individual, y la educación física, sin embargo, a diferencia de lo establecido en la revisión sistemática realizado por García (2016) no se observa una diferencia entre el deporte individual y de conjunto, esto puede deberse al nivel competitivo, ya que se ha observado que la satisfacción de la autonomía en deportes individuales, así como la satisfacción de la relación en deportes grupales va ligado al nivel competitivo de los deportistas, comparado con la muestra de este estudio que son deportistas colegiales, por lo que puede ser el carácter lúdico de los entrenamientos lo que esté modificando la percepción de la satisfacción de las NPB. En cuanto a la frustración de las NPB de forma individual, no se han encontrado estudios con los cuales se puedan realizar una comparación, sin embargo, al observar los resultados dados en donde la frustración de relación no se encuentra diferencia significativa entre ninguno de los grupos y la similitud de resultados entre la satisfacción de la autonomía y competencia con respecto a sus respectivas frustraciones, podemos suponer que el carácter lúdico y recreativo pudiese estar afectando los resultados, por tanto, será necesario realizar más estudios.

Pasando a la cohesión tarea y social en correspondencia a la naturaleza de la AF encontramos que, a diferencia de lo mostrado en otros estudios (Gómez-Acosta, Sierra-Barón, Aguayo, Matta, Solano, \& Oviedo, 2020) nuestros resultados no arrojan diferencia significativa entre ambos tipos de deporte (conjunto e individuales), esto puede deberse a que nuestros participantes pertenecen a un colegio de educación básica, por lo que la mayoría de los deportistas no sólo conviven dentro del equipo deportivo, si no que llevan clases escolares juntos, por lo que la cohesión, sobre todo a nivel social, puede estar siendo afectada por el tiempo y calidad de convivencia dentro de las clases curriculares y extracurriculares, por lo que estos resultados deben de ser tomados con cautela.

Con relación a la experiencia deportiva únicamente se vieron diferencias significativas dentro de cuatro variables; 1) clima empowering, donde los deportistas más jóvenes presentan niveles mayores, si bien no se encontraron estudios que analicen directamente el clima empowering en relacion a la experiencia deportiva, estos resultados va en línea con lo mostrado por Cordo-Cabal et al. (2019) quienes mencionan que a medida que se va incrementando la experiencia se empiezan a desarrollar climas motivacionales orientados a el ego; 2) satis- 
facción de las NPB y, específicamente la satisfacción de la autonomía y relación, en las que los individuos con menos de 4 años de experiencia reportan puntaciones superiores, esto va en contra de lo mostrado por ParraPlaza et al. (2018) en donde se relaciona la satisfacción de la autonomía y competencia con el incremento de experiencia en el deporte; 3) la cohesión tarea presenta mayor puntuación en el grupo de 4 años o menos, estos últimos resultados son innovadores y deben ser tomados con cautela debido a que no se ha encontrado en la literatura trabajos similares.

Las diferencias en resultados pueden deberse a que comúnmente se inicia a practicar un deporte por gusto o diversión dentro de un ambiente lúdico, pero a medida que se incremente el nivel competitivo y, por lo tanto, la experiencia, la importancia de ganar o demostrar mayores aptitudes que el compañero toma relevancia entrando a un ambiente mayormente competitivo (Cordo-Cabal et al., 2019); sin embargo, en nuestra muestra aquellos con mayor experiencia siguen estando en un nivel competitivo colegial, donde el componente lúdico sigue siendo predominante.

En el segundo objetivo del estudio, basado en las relaciones entre las variables se confirmaron las dos hipótesis planteadas. Primera, el clima empowering relaciona significativa y positivamente con la satisfacción de las NPB, lo cual coincide con lo dicho en el modelo establecido por Duda et al. (2018) y lo mostrado en otros artículos con diferentes poblaciones (Castillo et al., 2017; Erlend et al., 2018; Ramírez, 2020), de la misma forma se confirma que el clima empowering relaciona de forma negativa y significativa con la frustración de las NPB. Además, el clima empowering relacionó positiva y significativamente con ambas cohesiones (tarea y social), esto igualmente coincide con lo encontrado en otros artículos (Eys et al., 2009; Moehnke, 2019).

Para la segunda hipótesis, considerando la perspectiva obscura de la teoría, nuestros resultados igualmente apoyan lo establecido por Duda et al. (2018), siendo que el clima disempowering relacionó positiva y significativamente con la frustración de las NPB, esto también apoyado por otros artículos (Chu, 2018; Ramírez, 2020), y de forma contraria, se confirma la relación negativa y significativa con la satisfacción de las NPB, siendo esto similar a otro estudio (Erlend et al., 2018).

Estos resultados nos muestran como el promover un clima motivacional donde se apoye la autonomía, la socialización saludable, el trabajo en equipo y la mejora personal va a ayudar a que los deportistas desarrollen una salud psicológica por medio de la satisfacción de las NPB y que la unión y congruencia del equipo se fortifique. A su vez, los resultados nos evidencian que al desarrollar un clima motivacional donde no se respeten las diferencias individuales, juzgando el éxito únicamente por los resultados y con un estilo autoritario por parte del entrenador, va a impactar negativamente en el bienestar psicológico de nuestros deportistas.

Como limitaciones de este estudio es importante mencionar que los grupos poblacionales contrastados en cuestiones de número de muestra difieren en tamaño, por lo tanto, sería importante realizar investigaciones futuras donde esta característica se cumpla, además de seguir indagando sobre las diferencias con respecto a las variables psicológicas presentadas en diferentes grupos poblacionales, ya que se recalca la escasa información existente al respecto.

\section{Conclusión}

A manera de conclusión podemos evidenciar la relación positiva entre el clima empowering, la satisfacción de las NPB y ambas cohesiones (tarea y social), mostrando que, si el entrenador busca darle poder de decisión a sus deportistas, oportunidad de socializar de forma saludable y premia la mejora personal y el esfuerzo, es muy probable que el deportista desarrolle salud psicológica y se unifique el equipo deportivo.

Con respecto a las diferencias entre grupos, se observó que las características de la población y el ámbito en el que se desarrollan, pueden ser determinantes en la variabilidad de las respuestas psicológicas, por lo que, ante cualquier tipo de diagnóstico, intervención y evaluación, habrá que considerar tanto la relación entre las variables, como las características de la población con las que se trabajan.

\section{Referencias}

Amado,D.,Sánchez-Miguel,P.A.,Leo, F.M.,Sánchez-Oliva,D., \& García-Calvo,T. (2014). Diferencias de género en la motivación y percepción de utilidad del deporte escolar. Revista Internacional de Medicina y Ciencias de la Actividad Física y el Deporte, 14(56), 651-664.

Ames, C. (1992). Classrooms: Goals, Structures, and Student Motivation. Joumal of Educational Psychology, 84(3), 261-271. https://doi.org/10.1037/0022-0663.84.3.261

Appleton,P.R., Ntoumanis, N., Quested,E.,Viladrich, C., \& Duda, J.L.(2016). Initial validation of the coach-created Empowering and Disempowering Motivational Climate Questionnaire 
(EDMCQ-C).Psychology of Sport and Exercise, 22, 53- 65.https:/ /doi.org/10.1016/j.psychsport.2015.05.008

Bartholomew, K. J., Ntoumanis, N., Ryan, R. M., Bosch, J.A., \& Thøgersen-Ntoumani, C. (2011). Self-determination theory and diminishedfunctioning:The role of interpersonal control and psychological need thwarting. Personality and Social Psychology Bulletin, 37(11), 1459-1473. https://doi.org/ 10.1177/0146167211413125

Cantú-Berrueto, A., Castillo, I., López-Walle, J., Tristán, J., \& Balaguer,I.(2016). Estilo interpersonal del entrenador, necesidades psicológicas básicas y motivación: un estudio en futbolistas universitarios mexicanos. Revista Iberoamericana de Psicología del Ejercicio y el Deporte, 11(2), 263-270.

Carron, A.V., Widmeyer,W. N., \& Brawley, L. R. (1985). The Development of an Instrument toAssess Cohesion in Sport Teams:The GroupEnvironmentQuestionnaire. Journal of Sport Psychology, 7(3), 244-266. https://doi.org/10.1123/ jsp.7.3.244

Castillo, I., Ródenas, L. T.. , López-Walle, J. , \& Balaguer,I. (2017). Análisis preliminares de las propiedades psicométricas de la versión española del cuestionario del entorno deportivo para jóvenes en futbolistas. En J. M. López-Walle, R. Cuevas, P. Reynaga-Estrada,J.Tristán,A.Hernández-Mendo,I. Balaguer, y J.L.Duda(Ed.), Contribuciones de la Psicologia para una Red de Deporte de Calidad (pp. 27-35). México: UniversidadAutónomadeNuevo León.

Castillo, N., López-Walle, J. M.,Tomás, I. , \& Balaguer,I. (2017). Relación del clima empowering con la motivación autodeterminada a través de la satisfacción de las necesidades psicológicas básicas. Revista de Psicologia del Deporte, 26(3), 3339.

Castro-Sánchez, M.,Zurita-Ortega,F., \& Chacón-Cuberos, R. (2018). Clima motivacional en deportistas en función de la modalidad deportiva. SPORTTK-Revista EuroAmericana de Ciencias del Deporte, 8(1), 31-38. https://doi.org/10.6018/ sportk.362031

Chu,T.L. (2018). The Roles of Coaches, Peers, and Parents in High School Athletes'Motivational Processes:A Mixed-Methods Study. University of North Texas. https://doi.org/10.1177/ 1747954119858458

Cordo-Cabal,L., Gómez-López, M., Granero-Gallegos,A., \& Sánchez-Alcaraz, B. J. (2019). Relación del climamotivacional generado por el entrenador y las causas del éxito en jugadores de deportes de equipo. Influencia del género, la edad y la experiencia deportiva. Journal of Sport and Health Research, 11(2), 139-150.

Dobrijeviæ,G.,ĐorðeviæBoljanoviæ,J.,Alèakoviæ,S.,\&Lazareviæ, S. (2020). Perception of Cohesion in Interactive SportsTeams. Physical Education and Sport, 18(2), 431-438.https: / doi.org/ https: / /doi.org/10.22190/FUPES180831040D

Duda, J. L, Appleton, P. R., Stebbings, J., \& Balaguer, I. (2018). Towards more empowering and less disempowering environmentsin youth sport. En C. J. Knight, C. G. Harwood, \& D. Gould (Eds.) Sport Psychology forYoung Athletes (pp. 8193). New Yark: Taylor \& Francis Group. https: / / doi.org/ Https://doi.org/10.4324/9781315545202-8

Duda, J.L.; Appleton, P.R. (2016). Empowering and disempowering coaching behaviours: Conceptualisation, measurement considerations, and intervention implications. EnRaab, P.Wylleman, R. Seiler,A. M.Elbe,A.Harzigeorgiadis (Eds), Sport and Exercise Psychology Research: FromTheory to Practice (pp. 374-390). London, UK; Elsevier. https://doi.org/ 10.1016/B978-0-12-803634-1.00017-0

Erikstad, M. K., Martin,L. J., Haugen,T., \& Høigaard, R. (2018). Group cohesion, needs satisfaction, andself-regulated learning: A one-year prospective study of elite youth soccer players' perceptions of their club team. Psychology of Sport and Exercise, 39,171-178.https://doi.org/10.1016/ j.psychsport.2018.08.013

Erlend, B., Ivarsson, A., Haug, E. M., \& Ommundsen,Y. (2018). Youth Sport Coaches'Well-Being Across the Season: The Psychological Costs and Benefits of Giving Empowering and Disempowering Sports CoachingtoAthletes. International Sport Coaching Journal, 5, 124-135. https://doi.org/10.1123/ iscj.2017-0026

Eys, M., Loughead, T., Bray, S. R., \& Carron, A. V. (2009). Development of a Cohesion QuestionnaireforYouth:TheYouth SportEnvironment Questionnaire. Journal of Sport and Exercise Psychology, 31(3), 390-408. https://doi.org/10.1123/ jsep.31.3.390

Ferrnández, D. (2016). Relación entre cohesión y rendimiento en hombres y mujeres deportistas (tesis de grado). Universidad de Santiago de Compostela, España.

García, R. (2016). Revisión sobre las diferencias de las variables psicológicas en deportes individuales vs. deportes de equipo (tesis de grado). UniversidaddeCádiz, España. http:/ / hdl.handle.net/10498/ 18512

Gómez-Acosta,A., Sierra-Barón,W.,Aguayo,A. L., Matta, J. D., Solano,É.A., \& Oviedo,L.V.(2020). Caracterizaciónpsicosocial de deportistas y reconocimiento de laimportancia del psicólogodeportivo.PensamientoPsicológico, 18(2).https: / /doi.org/ https://doi.org/10.11144/Javerianacali.PPSI18-2.cpdr

González,L., Castillo,I., García-Merita, M., \& Balaguer,I.(2015). Apoyo a la autonomía, satisfacción de las necesidades psicológicas y bienestar: Invarianza de un modelo estructural en futbolistas y bailarines. Revista de Psicologia del Deporte, 24(1), 121-129. http: / / www.redalyc.org/ articulo.oa?id $=235139639014$

Gutiérrez-García,P., López-Walle, J.M., Tomás, I.,Tristán, J., \& Balaguer,I.(2019). Relación entre clima empowering y diversión en pitchers de béisbol: el papel moderador de la motivaciónautónoma. Cuadernos de Psicología del Deporte, 19(1), 166 177.https://doi.org/10.6018/cpd.353081

Hancox, J., Quested, E., Ntoumanis, N., \& Duda, J. L. (2017). 
Teacher-created social environment, basicpsychological needs, and dancers' affective states during class:A diary study. Personality and Individual Differences, 115, 137-143. https://doi.org/ 10.1016/j.paid.2016.03.033

Heuzé, J. P., Eys, M., Dubuc, M., Bosselut, G., \& Couture, R. (2018). Cohesion, psychological needs, andintrinsicmotivation in youthteam sport contexts. International journal of Sport Psychology, 49(1), 55-73. https://www.cabdirect.org/ cabdirect/abstract/20183113360

Leytón, M., García, J., Fuentes, J.P., \& Jiménez, R. (2018).Análisis de variables motivacionales y de estilos de vida saludables en practicantes de ejercicio físico en centros deportivos enfunción del género. Retos, 34, 166-171. https://doi.org/ 10.47197/retos.v0i34.58281

López-Walle, J., Balaguer, I., Castillo, I., \& Tristán, J. (2012). Autonomy Support, Basic Psychological NeedsandWell-Being in MexicanAthletes. The Spanish Journal of Psychology, 15(3), 1283-1292. https://doi.org/10.5209/ rev_sjop.2012.v15.n3.39414

Mars, L., Castillo, I., López-Walle, J., \& Balaguer, I. (2017). Estilo controlador del entrenador, frustración de las necesidades y malestar en futbolistas. Revista de Psicología del Deporte, 26(2), 119-124. http://www.redalyc.org/ articulo.oa?id=235152045017

McAuley, E., Duncan,T., \&Tammen,V.V.(1989). Psychometric Propertiesof theIntrinsicMotivationInventoryinaCompetitive Sport Setting - A Confirmatory Factor-Analysis. Research Quarterly for Exercise and Sport, 60(1), 48-58. https: / / doi.org/ 10.1080/02701367.1989.10607413

Moehnke, H. J. (2019). A United Front: Coach and Teammate Motivational Climate andTeam Cohesion among Adolescent Female Athletes (tesis de maestría).. University of Minnesota, EUA. https: / /doi.org/10.22201/fq.18708404e.2004.3.66178

Mosqueda, S., López-Walle, J. M., Gutiérrez-García, P., GarcíaVerazaluce, J., \&Tristán,J.(2019). Autonomous Motivationas a Mediator Between anEmpowering Climate and Enjoyment in Male Volleyball Players. Sports, 7 (153), 2-8 . https: / / doi.org/10.3390/sports7060153

NascimentoJr,J.R.A., Silva,A.A., Granja, C.T.L., Oliveira, D.V., \& Fortes,L.deS. (2019). Do sporting experiences predict team cohesion in youth athletes? Cuadernos de Psicología del Deporte, 19(3), 102-112.https://doi.org/10.6018/cpd.365201

Nascimiento Jr,J.R.A., Nickenig, J. R., Lavallee, D., Codonhato, R., Do Nascimiento, J. B., \& Flores, L. (2017).The mediating role of basic needs satisfaction on the relationship of perfectionism traits and team cohesion among elite futsal athletes. International Journal of Sport Psychology, 48, 591-609. https: / /doi.org/10.7352/IJSP

Nicholls, J.G. (1984). Achievement motivation: Conceptions of ability, subjective experience, task choice, and performance. Psychological Review, 91(3), 328-346. https://doi.org/ 10.1037/0033-295X.91.3.328
Papalia, D. E., Olds, S.W., \& Feldman, R. D. (2009). Desarrollo Humano. México: McGraw Hill. Recuperado de http: / / hdl.handle.net/10637/2315

Parra-Plaza,F. J.,Vílchez-Conesa, M. delP., \& DeFrancisco-Palacios, C. (2018). La satisfacción de las necesidades psicológicas básicas en función de la formación y experiencia del deportista: características sociodeportivas. Revista de Psicología y Educación-Journal of Psychology and Education, 13(2), 113-123. https: / /doi.org/10.23923/rpye2018.01.162

Pavón,A., \& Moreno, J.A. (2008). Actitud de los universitarios antela prácticafísicodeportiva: diferencias por géneros. Revista dePsicología del Deporte, 17(1), 7-23.

Ramírez, J. (2020). Climas empoweringy disempowering,necesidades psicológicas e intención de práctica deportiva futura en jóvenes deportistas (tesis de maestrai). Universidad Autónoma de Nuevo León, México.

Reinboth, M.,\&Duda,J.L.(2006). Perceivedmotivational climate, need satisfaction and indices of well-being in team sports: A longitudinal perspective. Psychology of Sport and Exercise, 7(3), 269-286. https://doi.org/doi.org/10.1016/ j.psychsport.2005.06.002

Richer, S., \&Vallerand, R. (1998). Construction et validation de I'Echelle dusentiment d'appartenance social. Revue Européenne de PsychologieAppliqué, 48, 129-137.

Romina, R. (2019). Análisis del clima motivacional y su relación con la intención de abandono en los nadadores de Zona Sur de Buenos Aires (tesis de grado). UniversidadAbierta Interamericana,Argentina.

Ryan, R. M., yDeci,E.L.(2000). Self-determinationTheoryand thefacilitation of intrinsicmotivation, socialdevelopmentand well-being. American Psychologist, 55, 1,68-78. http://doi.org/ 10.1037110003-066X.55.1.68

Ryan, R. M., y Deci, E. L. (2017). Self-determination theory: Basic Psychological Needs in Motivation Development andWellness. New York, NY,USA: Guilford Publishing.

Salazar-Ayala, C.M., \& Gastélum-Cuadras, G. (2020).Teoría dela Autodeterminación en el contexto de educación física: Una revisión sistemática. Retos, 38, 834 844. https: / / doi.org/ 10.47197/retos.v38i38.72729

SurveyMonkey.(n.d.). SurveyMonkey Inc. es.surveymonkey.com Torregrosa, M.,Viladrich,C., Ramis,Y.,Azócar,F., Latinjak,A.T., \& Cruz,J.(2011). Efectos en lapercepción del dimamotivacional generado por los entrenadores y compañeros sobre la diversión y el compromiso. Diferencias en función de género. Revista de Psicologia del Deporte, 20(1), 243-255. 